Mathematical Problems of Computer Science 52, 30-42, 2019.

UDC 519.1

\title{
Rumor Spreading and Invasion Percolation
}

\author{
Suren S. Poghosyan and Vahagn S. Poghosyan \\ Institute for Informatics and Automation Problems of NAS RA \\ e-mail: psuren55@yandex.ru,povahagn@gmail.com
}

\begin{abstract}
We study the models of rumor spreading and invasion bond percolation aimed at the revelation of possible connections between them. Rumor spreading model describes the dissemination of a rumor due to the periodical repetition of sequential phone calls, whereas the invasion bond percolation refers to the spread of liquid in the porous environment. During a round of the rumor spreading, each node performs a call only once, meanwhile transmitting all the information that it knows at the moment of the call to its neighbors. The rumor reaches the receiver node during one round if there is a chain of successive calls between the source of the rumor and that node. The sequence of calls is taken uniformly at random from the set of all possible sequences (permutations of nodes). We compare the propagation of the rumor spreading with the invasion bond percolation in order to put forth necessary improvements of the percolation rules to map one model onto another, and vice versa.
\end{abstract}

Keywords: Gossip problem, Information dissemination, Invasion percolation.

\section{Introduction and Models}

The spreading of rumors and invasion percolation are core problems in two different fields: the theory of dissemination of information [1]-[17] and the theory of flow in a porous media [18]-[23]. Both problems concern with the stochastic growth of clusters: the cluster of informed individuals in the first case and the cluster of invaded sites in the second one. Here, we consider two models typical for each problem and establish a link between them.

Among the variety of formulations of the first problem, we choose the random phone call model [19] defined on the so called communication graph $G(V, E)$ with the set of vertices $V$ and the set of edges $E$. Vertices $V$ denote the set of players, the edges $E$ denote possible connections between the players by phone calls. Whenever a connection is established, the rumor can be transmitted from one player (if she holds the rumor) to another player (if she does not hold the rumor yet). In [19], the authors considered the model where the players communicate in parallel during communication rounds. It means that any information received in a round cannot be forwarded to another player in the same round. The aim is to spread all rumors from all players to others. The problem was to find an algorithm using only $O(\ln |V|)$ rounds and $O(|E| \ln \ln |E|)$ calls. 
In this paper, we concentrate on kinetics of the rumor propagation on the communication graph $G(V, E)$. To this end, we investigate the effect of sequential communications when a rumor can be translated through a chain of random calls during one round. Correspondingly, each round in our model is a finite time interval containing all possible connections $e_{i} \in E, i=$ $1,2, \ldots,|E|$, which happen exactly once at moments $t(i)$, where $t(i) \equiv t\left(e_{i}\right)$ is the moment of connection $e_{i}$ in the current round. We assume that all moments $t(i), i=1,2, \ldots,|E|$ are distinct and uniformly distributed between the beginning and the end of each round. The effect of sequential communications leads to a specific pattern of the cluster growth, which is similar to the invasion percolation. However, the rules of growth of the clusters of informed players and invaded sites in the percolation problem are not completely identical.

Let $v^{*} \in V$ be a fixed player who is the origin of the rumor and $P=\left(p_{1}, p_{2}, \ldots, p_{|E|}\right)$ is a permutation of numbers $(1,2, \ldots,|E|)$ such that $t\left(p_{1}\right)<t\left(p_{2}\right)<\cdots<t\left(p_{|E|}\right)$ in the first round. Denoting by $e_{i j} \in E$ the edge connecting adjacent vertices $i \in V$ and $8 j \in V$, we define an ordered path between the vertices $i_{1} \in V$ and $i_{k} \in V$ as the sequence of connections $e_{i_{1} i_{2}}, e_{i_{2} i_{3}}, \ldots, e_{i_{k-1} i_{k}}$. The path between $i_{1}$ and $i_{k}$ conducts the rumor if $t\left(e_{i_{1} i_{2}}\right)<t\left(e_{i_{2} i_{3}}\right)<$ $\cdots<t\left(e_{i_{k-1} i_{k}}\right)$. Given the permutation of calls $P$, we will say that the rumor started from $v^{*}$ reaches player $v \in V$ in the first round if there exists a conducting path between $v^{*}$ and $v$.

The subset $V_{1} \subset V$ of vertices reachable from $v^{*}$ during the first round represents the set of players getting informed due to permutation of calls $P$. The quantity of interest is the number of informed players $\left|V_{1}\right|_{P}$ and its average taken over all permutations $\left\langle\left|V_{1}\right|\right\rangle=$ $\sum_{P}\left|V_{1}\right|_{P} \operatorname{Prob}\left|V_{1}\right|_{P}$. A conducting path between two arbitrary vertices is not necessarily selfavoiding. Indeed, the path may contain one or more loops, which appear if both adjacent vertices $i$ and $j$ in connection $e_{i j}$ belonging to the path are already informed before the moment $t\left(e_{i j}\right)$. We can coin such a connection as non-informative in contrast to a informative connection that conducts the rumor. The exclusion of non-informative connections from all conducting paths removes all possible loops. Then, the remaining informative connections form a connected graph, which is a tree spanning the subset of informed players $V_{1}$ and is rooted at $v^{*}$. By definition, the first round is complete if all possible connections $e_{i} \in E, i=$ $1,2, \ldots,|E|$ happened exactly once. Alternatively, we can say that the round is complete only if subset $V_{1}$ contains all possible vertices reachable from $v^{*}$ for the given permutation $P$.

When the first round is over, the next rounds start consecutively with the same sequence of connections $t(i), i=1,2, \ldots,|E|$. Players receiving the rumor in the first round can serve as origins of the same rumor for the second round. As above, the rumor is spreading along the conducting paths started at the boundary vertices of subset $V_{1}$. (As usual, we call a vertex $v \in V$ the boundary vertex of a subset $V_{1} \subset V$ if $v \in V_{1}$ and at least one of the nearest neighbors of $v$ in graph $G(V, E)$ does not belong to $\left.V_{1}\right)$. The collection of informative connections in the second round forms a graph, which is a forest with roots at the boundary vertices of $V_{1}$. The forest spans the subset of players $V_{2}$ being informed during the second complete round.

Continuing the process round by round, we obtain a sequence of subsets of players $V_{i}, i=$ $1,2, \ldots$ characterized by the size $\left|V_{i}\right|_{P}$ and the average size $\left\langle\left|V_{i}\right|\right\rangle$ for each round and by the average correlations between the rounds. The process is finished when all players $V$ become informed with the rumor. The question is the average number of rounds needed for the total spreading of the rumor.

The associated problem, invasion percolation, has many formulations as well [20]. We 
choose from them a popular version, namely, the invasion bond percolation model [21]. Similar to the first problem, we start with the graph $G(V, E)$ with sets of vertices and edges $V$ and $E$. We assign random numbers $p_{i j} \in[0,1]$ to each edge $e_{i j} \in E$. Invasion percolation is a process of successive occupation of the vertices and edges of $G(V, E)$. At the first step, one occupies a randomly chosen vertex $v^{*} \in V$. Then, one considers all edges adjacent to $v^{*}$ and occupies the edge with the smallest $p_{i j}$ together with the second vertex belonging to it. At subsequent steps, one finds the empty edge with the smallest $p_{i j}$ connected to the occupied vertices, and checks whether this edge connects two occupied vertices or not. In the former case, the edge is called trapped since it is situated between two occupied vertices. If the edge is not trapped, it becomes occupied together with the empty vertex it connects with the occupied cluster. In this way, one constructs a graph of occupied edges and vertices $T \subset G(V, E)$, which is a tree spanning the cluster of occupied vertices at the given stage.

The described model was introduced by Barabasi [22] and called the invasion bond percolation model with a local trapping rule to distinguish it from the ordinary invasion bond percolation where the trapped edges can be occupied as well as the non-trapped ones. Barabasi proved that the trapping rule does not affect the scaling and dynamic properties of growing clusters of occupied vertices and,therefore, the two models belong to the same universality class. Moreover, the construction of tree $T$ in the Barabasi's model coincides with the Prim's algorithm for finding the shortest spanning tree of a weighted random graph [23]. In the next section, we consider a modification of the Prim's algorithm to map it into the model of transmission of rumors. The subsequent sections will be devoted to the exact solutions of the latter model for simplified graphs $G(V, E)$ and the comparison of cluster growth in models of transmission of rumors and the invasion bond percolation.

\section{The Modified Model of Invasion Percolation}

The main difference between the models of transmission of rumors and the invasion bond percolation is the monotonic character of the invasion process, which is not subdivided into finite time periods appearing in the propagation of rumors. The invasion process continues permanently and stops when all vertices of graph $G(V, E)$ become occupied. The propagation of rumors is intermitted at the end of each complete round when all players reachable for the given permutation of calls become informed. To eliminate the difference, we introduce a modified model of invasion percolation (or a modified Prim's algorithm).

As before, we begin with the graph $G(V, E)$ and the set of random weights $p(i, j) \in[0,1]$ assigned to each edge $e_{i j} \in E$. Again, we choose a vertex $v^{*} \in V$ and consider it as the starting point of a growing cluster. The cluster grows by successive adding new occupied edges. The occupation of edge $e_{i j}$ means the occupation of adjacent vertices $i$ and $j$ as well. The addition process yields two restrictions:

(a) Each new edge we are going to occupy connects two vertices, one of which belongs to the cluster and the other does not.

This rule guaranties that the growing cluster is a tree. Of two connected edges of the tree, we call a predecessor the edge that is closer to the starting point. The other edge is the successor.

(b) Each new occupied edge being a successor, has the weight larger than its predecessor.

The edges yielding both rules (a) and (b) are called eligible for the growth. The modified model of invasion percolation is defined by the following steps. The first step is the occupation of the starting vertex $v^{*}$. Given a connected cluster of edges and vertices $C(i)$ obtained after 
$i$ steps, $i=1,2, \ldots$, we select the set of edges $E(i)$ eligible for the growth (for edges adjacent to the starting point, the rule (b) is omitted). Choose in the set $E(i)$ the edge $e_{i j}$ with minimal weight $p(i, j)$ and add it to the cluster of occupied edges and vertices. In this way, we obtain the cluster $C(i+1)$.

If, after some number of steps $i_{1}$, there are no edges eligible for growth, we fix the cluster $C\left(i_{1}\right)$ and start the next round of growth. To this end, we take the same set of random weights $p(i, j) \in[0,1]$ and consider the boundary vertices of the set $C\left(i_{1}\right)$ as the starting points for the next round. The cluster of edges and vertices obtained after $i$-th step of the second round is denoted by $C\left(i_{1}, i\right)$. For each step of the second round, we define the set $E\left(i_{1}, i\right)$ of edges eligible for growth and choose among them the edge $e_{i j}$ with minimal weight $p(i, j)$. The second round continues during a number of steps $i_{2}$ until one is able to find edges eligible for growth. The set of edges and vertices occupied during the second round is $C\left(i_{1}, i_{2}\right)$. The process proceeds round by round until the set of vertices in $C\left(i_{1}, i_{2}, \ldots, i_{\max }\right)$ coincides with that in $G(V, E)$.

We get a modified model of invasion bond percolation displaying an intermittence of the invasion process. In order to get the correspondence between the introduced model and the random call model, we restrict the length of each round in the latter model to the unit time interval. Then, we can identify the moments of calls $t(i), i=1,2, \ldots,|E|$ with the random numbers $p_{i j} \in[0,1]$ assigned to each edge $e_{i j} \in E$. The condition that all $p_{i j}$ are distinct is not essential since $p_{i j}$ are continuous variables. The condition of completeness of each round is identical for both models if one takes into account the ability of a cluster to grow during the round.

\section{The One-dimensional Lattice}

In this section, we illustrate the considered models with a simplified example, where the communication graph $G(V, E)$ is a finite one-dimensional lattice. The set of vertices $V \subset \mathbb{Z}$ representing the players consists of $L+1$ lattice points $i \in V, i=1,2, \ldots, L+1$. The set of edges $E$ represents $L$ connections $e_{j} \in E$ between the neighboring vertices $j$ and $j+1$, $j=1,2, \ldots, L$.

The origin of the rumor is the first player $i=1$, and $t(j) \equiv t\left(e_{j}\right)$ are the moments of calls used by the connections $e_{j}, j=1,2, \ldots, L$. The order of calls corresponds to the permutation $P=\left(p_{1}, p_{2}, \ldots, p_{L}\right)$ such that $t\left(p_{1}\right)<t\left(p_{2}\right)<\cdots<t\left(p_{L}\right)$. The rumor reaches the player $k_{1}+1$ during the first round and does not propagate further if

$$
t(1)<t(2)<\ldots<t\left(k_{1}\right), t\left(k_{1}\right)>t\left(k_{1}+1\right) .
$$

Consider $k_{1}+1$ numbers $t(1), t(2), \ldots, t\left(k_{1}+1\right)$. Among $\left(k_{1}+1\right)$ ! permutations of these numbers, only one is ordered as

$$
t(1)<t(2)<\ldots<t\left(k_{1}\right)<t\left(k_{1}+1\right)
$$

with probability $1 /\left(k_{1}+1\right)$ !. The order $(2)$ can be broken and converted into (1) in $k_{1}$ ways by replacing any of $k_{1}$ first numbers with $t\left(k_{1}+1\right)$. Therefore, the probability of order (1) is $k_{1}$ times greater than that of $(2)$ and we get

$$
\operatorname{Prob}\left(k_{1}\right)=\frac{k_{1}}{\left(k_{1}+1\right) !} .
$$


The rumor reaches the player $k_{1}+1$ during the first round and the player $k_{1}+k_{2}+1$ during the second round but does not propagate further if

$$
t(1)<t(2)<\cdots<t\left(k_{1}\right) ; \quad t\left(k_{1}+1\right)<t\left(k_{1}+2\right)<\cdots<t\left(k_{1}+k_{2}\right)
$$

but

$$
t\left(k_{1}\right)>t\left(k_{1}+1\right) ; \quad t\left(k_{1}+k_{2}\right)>t\left(k_{1}+k_{2}+1\right)
$$

To find the probability $\operatorname{Prob}\left(k_{1}, k_{2}\right)$, one has to exclude $\operatorname{Prob}\left(k_{1}+k_{2}\right)$ from the product of two probabilities $1 / k_{1}$ ! and $\operatorname{Prob}\left(k_{2}\right)$ :

$$
\operatorname{Prob}\left(k_{1}, k_{2}\right)=\frac{k_{2}}{k_{1} !\left(k_{2}+1\right) !}-\frac{k_{1}+k_{2}}{\left(k_{1}+k_{2}+1\right) !} .
$$

The calculation of further probabilities $\operatorname{Prob}\left(k_{1}, k_{2}, \ldots, k_{n}\right)$ is a direct application of the inclusion-exclusion principle. For instance,

$$
\begin{aligned}
& \operatorname{Prob}\left(k_{1}, k_{2}, k_{3}\right)=k_{3} /\left(k_{1} ! k_{2} !\left(1+k_{3}\right) !\right)-k_{3} /\left(\left(k_{1}+k_{2}\right) !\left(1+k_{3}\right) !\right)- \\
& \quad\left(k_{2}+k_{3}\right) /\left(k_{1} !\left(1+k_{2}+k_{3}\right) !\right)+\left(k_{1}+k_{2}+k_{3}\right) /\left(1+k_{1}+k_{2}+k_{3}\right) !
\end{aligned}
$$

Knowing the probabilities $\operatorname{Prob}\left(k_{1}, k_{2}, \ldots, k_{i}\right)$, we can calculate the expected values $\left\langle k_{i}\right\rangle$ in the limit $L \rightarrow \infty$ :

$$
\left\langle k_{i}\right\rangle=\sum_{k_{1}=1, \ldots, k_{i}=1}^{\infty} k_{i} \operatorname{Prob}\left(k_{1}, k_{2}, \ldots, k_{i}\right)
$$

The first several results are:

$$
\begin{gathered}
\left\langle k_{1}\right\rangle=e-1 \\
\left\langle k_{2}\right\rangle=e^{2}-2 e \\
\left\langle k_{3}\right\rangle=\frac{3 e}{2}-3 e^{2}+e^{3} \\
\left\langle k_{4}\right\rangle=-\frac{2 e}{3}+4 e^{2}-4 e^{3}+e^{4} \\
\left\langle k_{5}\right\rangle=\frac{5 e}{24}-\frac{10 e^{2}}{3}+\frac{15 e^{3}}{2}-5 e^{4}+e^{5}
\end{gathered}
$$

the numerical values of which are:

$$
\begin{aligned}
\left\langle k_{1}\right\rangle & =1.718281828 \ldots \\
\left\langle k_{2}\right\rangle & =1.952492442 \ldots \\
\left\langle k_{3}\right\rangle & =1.995791369 \ldots \\
\left\langle k_{4}\right\rangle & =2.000038850 \ldots \\
\left\langle k_{5}\right\rangle & =2.000057578 \ldots
\end{aligned}
$$


One can expect that $\left\langle k_{n}\right\rangle \rightarrow 2$ when $n \rightarrow \infty$. To prove this conjecture, it is sufficient to find the generating function (see Appendix )

$$
R(x)=\sum_{m=1}^{\infty}\left\langle k_{m}\right\rangle x^{m}=\frac{1-x}{1-x \exp (1-x)}
$$

and verify that

$$
R(x) \sim 2 /(1-x)+O(x-1)
$$

in the vicinity of the point $x=1$.

\section{Conclusion}

In our works [24]-[29], toppling of grains at nodes (graph vertices in Abelian sandpile model) was interpreted as a transmission of the full information accumulated in a vertex, activated at the moment, to all its neighbors ( $k$-broadcast). Taking into account that activation of vertices is performed according to a predefined random order, and a single time tact is defined to be a discrete time interval (equal to the number of vertices) of activation of all the vertices, it is necessary to estimate the average number of tacts required for the full information exchange/transmission.

The exact formula for the average number of tacts for $(n, n)$ lattices has not yet been derived. Nevertheless, based on the required number of experimental data, statistical curves of the main characteristics have been obtained, the study of which made it possible to set up a hypothesis (research methods and results are not included in this paper) that the average number of tacts is $0.25 \mathrm{n}$.

Study of the models of rumor spreading and invasion bond percolation, aimed at the revelation of possible connections between them, is performed. It is shown that the rumor reaches the receiver node during one round if there is a chain of successive calls between the source of the rumor and that node. Given that the sequence of calls is taken uniformly at random from the set of all possible sequences, analytical properties of information dissemination have been investigated.

The results obtained make it possible to improve the percolation rules aimed at mapping one model onto another, and vice versa. Future work will be dedicated to the comparative analysis between rumor spreading and invasion bond percolation models.

\section{Appendix}

We define a set of all partitions $\mathbb{P}\left(\left\{k_{1}, k_{2}, \ldots, k_{n}\right\}\right)$ of a set of $n$ integers $\left\{k_{1}, k_{2}, \ldots k_{n}\right\}$ as a set of the following $2^{n-1}$ subsets

$$
\begin{gathered}
\mathbb{P}\left(\left\{k_{1}\right\}\right)=\left\{\left\{k_{1}\right\}\right\} \\
\mathbb{P}\left(\left\{k_{1}, k_{2}\right\}\right)=\left\{\left\{\left\{k_{1}\right\},\left\{k_{2}\right\}\right\},\left\{\left\{k_{1}, k_{2}\right\}\right\}\right\}
\end{gathered}
$$

In general, recursively, if the set of $m$ subsets of $\left\{k_{1}, k_{2}, \ldots, k_{n}\right\} S_{i}, i=1,2, \ldots, m$, is a partition

$$
\left\{S_{1}, S_{2}, \ldots, S_{m}\right\} \in \mathbb{P}\left(\left\{k_{1}, k_{2}, \ldots, k_{n}\right\}\right)
$$


then

$$
\begin{aligned}
\left\{S_{1}, S_{2}, \ldots, S_{m},\left\{k_{n+1}\right\}\right\} & \in \mathbb{P}\left(\left\{k_{1}, k_{2}, \ldots, k_{n+1}\right\}\right) \text { and } \\
\left\{S_{1}, S_{2}, \ldots, S_{m-1}, S_{m} \cup\left\{k_{n+1}\right\}\right\} & \in \mathbb{P}\left(\left\{k_{1}, k_{2}, \ldots, k_{n+1}\right\}\right)
\end{aligned}
$$

are also partitions. Each of $S_{i}, i=1,2, \ldots, m$ in (4) and (5) is a set of $k$ integers $S=$ $\left\{l_{1}, l_{2}, \ldots, l_{k}\right\}$, where $l_{i} \in \mathbb{N}, i=1,2, \ldots, k$. We denote $|S|=k$ and $\|S\|=l_{1}+l_{2}+\cdots+$ $l_{k}$. Then, (using the inclusion-exclusion principle), we obtain a sum over all elements of $\mathbb{P}\left(\left\{k_{1}, k_{2}, \ldots, k_{n}\right\}\right)$ :

$$
\operatorname{Prob}\left(\mathrm{k}_{1}, \mathrm{k}_{2}, \ldots, \mathrm{k}_{\mathrm{n}}\right)=\sum_{\mathrm{m}=1}^{\mathrm{n}} \sum_{\left.\left.\left.\left\{\mathrm{S}_{1}, \mathrm{~S}_{2}, \ldots, \mathrm{S}_{\mathrm{m}}\right\} \in \mathbb{P}\left(\{\urcorner_{\nVdash},\right\urcorner_{\not}, \ldots,\right\urcorner_{\ltimes}\right\}\right)} \frac{(-1)^{\mathrm{n}+\mathrm{m}}}{\left\|\mathrm{S}_{1}\right\| !\left\|\mathrm{S}_{2}\right\| ! \cdots\left\|\mathrm{S}_{\mathrm{m}-1}\right\| !} \frac{\left\|\mathrm{S}_{\mathrm{m}}\right\|}{\left(\left\|\mathrm{S}_{\mathrm{m}}\right\|+1\right) !} .
$$

Therefore, the average value of $k_{n}$ is

$$
\begin{aligned}
\left\langle k_{n}\right\rangle & =\sum_{k_{1}=1}^{\infty} \sum_{k_{2}=1}^{\infty} \ldots \sum_{k_{n}=1}^{\infty} k_{n} \operatorname{Prob}\left(\mathrm{k}_{1}, \mathrm{k}_{2}, \ldots, \mathrm{k}_{\mathrm{n}}\right) \\
& =\sum_{m=1}^{n} \sum_{\left\{S_{1}, S_{2}, \ldots, S_{m}\right\} \in \mathbb{P}(\{\nVdash, \nvdash, \ldots, \ltimes\})}(-1)^{n+m} \alpha_{\left|S_{1}\right|} \alpha_{\left|S_{2}\right|} \cdots \alpha_{\left|S_{m}\right|},
\end{aligned}
$$

where $\alpha_{n}, n>0$ is the $n$-fold sum

$$
\alpha_{n}=\sum_{k_{1}=1}^{\infty} \sum_{k_{2}=1}^{\infty} \cdots \sum_{k_{n}=1}^{\infty} \frac{1}{\left(k_{1}+k_{2}+\cdots+k_{n}\right) !}
$$

To calculate ( 7$)$ with $n=1,2, \ldots$, we introduce the generating function

$$
f_{n}(x)=\sum_{k_{1}=1}^{\infty} \sum_{k_{2}=1}^{\infty} \cdots \sum_{k_{n}=1}^{\infty} \frac{x^{k_{1}+k_{2}+\cdots+k_{n}}}{\left(k_{1}+k_{2}+\cdots+k_{n}\right) !}
$$

The derivative of $f_{n}(x)$ can be expressed in the following way

$$
\begin{aligned}
\frac{d f_{n}(x)}{d x} & =\sum_{k_{1}=1}^{\infty} \sum_{k_{2}=1}^{\infty} \cdots \sum_{k_{n}=1}^{\infty} \frac{x^{k_{1}+k_{2}+\cdots+k_{n}-1}}{\left(k_{1}+k_{2}+\cdots+k_{n}-1\right) !} \\
& =\sum_{k_{1}=1}^{\infty} \sum_{k_{2}=1}^{\infty} \cdots \sum_{k_{n-1}=1}^{\infty} \sum_{k_{n}=0}^{\infty} \frac{x^{k_{1}+k_{2}+\cdots+k_{n}}}{\left(k_{1}+k_{2}+\cdots+k_{n}\right) !} \\
& =f_{n}(x)+f_{n-1}(x) .
\end{aligned}
$$

We multiply both sides of the recursive differential equation (8) by $z^{n}$ and take the sum over $n=2,3, \ldots$. Introducing the generating function of the sequence of functions $f_{n}(x)$ :

$$
F(z, x)=\sum_{n=1}^{\infty} f_{n}(x) z^{n}
$$


and taking into account that

$$
f_{1}(x) \equiv \sum_{k_{1}=1}^{\infty} \frac{x^{k_{1}}}{k_{1} !}=e^{x}-1
$$

we obtain a differential equation for $F(z, x)$ :

$$
\frac{\partial F(z, x)}{\partial x}=(z+1) F(z, x)+z
$$

with initial conditions $F(z, 0)=0$. The solution of $(9)$ is

$$
F(z, x)=\frac{z}{z+1}\left(e^{x(z+1)}-1\right),
$$

which can be expanded over variables $x$ and $z$ :

$$
F(z, x)=\sum_{k=1}^{\infty} \frac{z(z+1)^{k-1}}{k !} x^{k}=\sum_{k=1}^{\infty} \sum_{n=1}^{k} \frac{x^{k} z^{n}}{(n-1) !(k-n) ! k}=\sum_{n=1}^{\infty} \sum_{k=n}^{\infty} \frac{x^{k} z^{n}}{(n-1) !(k-n) ! k} .
$$

Therefore,

$$
f_{n}(x)=\frac{1}{(n-1) !} \sum_{k=0}^{\infty} \frac{x^{n+k}}{k !(n+k)}=\frac{1}{(n-1) !}{ }_{0}^{x} \tau^{n-1} e^{\tau} \tau
$$

Thus, we obtain an exact expression for $\alpha_{n}$

$$
\alpha_{n}=f_{n}(1)=(-1)^{n}+e \sum_{p=1}^{n} \frac{(-1)^{n+p}}{(p-1) !}
$$

which allows one to calculate the values of $\alpha_{n}$ for various $n$ :

$$
\begin{array}{ccc}
\alpha_{1}=e-1, & \alpha_{2}=1, & \alpha_{3}=\frac{1}{2}(e-2), \\
\alpha_{4}=\frac{3-e}{3}, & \alpha_{5}=\frac{1}{8}(3 e-8), & \alpha_{6}=\frac{1}{30}(30-11 e) .
\end{array}
$$

Using the fact that

$$
\frac{1}{e}=\sum_{p=0}^{\infty} \frac{(-1)^{p}}{p !}
$$

we obtain an alternative representation of $\alpha_{n}$ :

$$
\alpha_{n}=e \sum_{p=0}^{\infty} \frac{(-1)^{p}}{(p+n) !}=\frac{e}{n !}-\frac{e}{(n+1) !}+\frac{e}{(n+2) !}-\cdots
$$

Note that

$$
\frac{e}{n !}-\frac{e}{(n+1) !}<\alpha_{n}<\frac{e}{n !}
$$

i.e.,

$$
\frac{n}{n+1} \frac{e}{n !}<\alpha_{n}<\frac{e}{n !}
$$


Therefore, the asymptotics of $\alpha_{n}$ for large $n$ is

$$
\alpha_{n} \simeq \frac{e}{n !}, \quad n \gg 1 \text {. }
$$

Now, we can rewrite the expression (6) for $\left\langle k_{n}\right\rangle$ in the following form

$$
\left\langle k_{n}\right\rangle=\sum_{\sigma_{1}=0}^{\infty} \sum_{\sigma_{2}=0}^{\infty} \cdots \sum_{\sigma_{n}=0}^{\infty} \delta\left(\sum_{i=1}^{n} i \sigma_{i}, n\right) \prod_{i=1}^{n}\left((-1)^{1+i} \alpha_{i}\right)^{\sigma_{i}} \frac{\left(\sum_{i=1}^{n} \sigma_{i}\right) !}{\sigma_{1} ! \sigma_{2} ! \cdots \sigma_{n} !} .
$$

The corresponding generating function is

$$
R(x)=1+\sum_{n=1}^{\infty}\left\langle k_{n}\right\rangle x^{n}=\sum_{\sigma_{1}=0}^{\infty} \sum_{\sigma_{2}=0}^{\infty} \cdots\left(\prod_{i=1}^{\infty} \frac{\left((-1)^{1+i} \alpha_{i}\right)^{\sigma_{i}}}{\sigma_{i} !}\right)\left(\sum_{i=1}^{\infty} \sigma_{i}\right) ! x^{\sum_{i=1}^{\infty} i \sigma_{i}} .
$$

To calculate $R(x)$, we introduce another generating function

$$
D(x, z)=\sum_{\sigma_{1}=0}^{\infty} \sum_{\sigma_{2}=0}^{\infty} \cdots\left(\prod_{i=1}^{\infty} \frac{\left((-1)^{1+i} \alpha_{i}\right)^{\sigma_{i}}}{\sigma_{i} !}\right) z^{\sum_{i=1}^{\infty} \sigma_{i}} x^{\sum_{i=1}^{\infty} i \sigma_{i}},
$$

which can be rewritten as

$$
\begin{aligned}
D(x, z) & =\prod_{i=1}^{\infty}\left(\sum_{\sigma=0}^{\infty} \frac{\left((-1)^{1+i} \alpha_{i}\right)^{\sigma} z^{\sigma} x^{i \sigma}}{\sigma !}\right) \\
& =\prod_{i=1}^{\infty} \exp \left\{(-1)^{1+i} \alpha_{i} x^{i} z\right\} \\
& =\exp \left\{z \sum_{i=1}^{\infty}(-1)^{1+i} \alpha_{i} x^{i}\right\}
\end{aligned}
$$

The function $D(x, z)$ is the series

$$
D(x, z)=\sum_{p=0}^{\infty} \frac{G(x)^{p}}{p !} z^{p}
$$

where

$$
\begin{aligned}
G(x) & =\sum_{i=1}^{\infty}(-1)^{1+i} \alpha_{i} x^{i} \\
& =\sum_{i=1}^{\infty}\left(-1+e \sum_{j=1}^{i} \frac{(-1)^{j-1}}{(j-1) !}\right) x^{i} \\
& =\frac{x}{x-1}+e \sum_{j=1}^{\infty}\left(\frac{(-1)^{j-1}}{(j-1) !} \sum_{i=j}^{\infty} x^{i}\right) \\
& =\frac{x}{x-1}+\frac{e}{1-x} \sum_{j=1}^{\infty} \frac{(-1)^{j-1} x^{j}}{(j-1) !} \\
& =\frac{x}{x-1}\left(1+e^{1-x}\right)
\end{aligned}
$$


On the other hand, the function $R(x)$ can be expressed by $G(x)$ :

$$
\begin{gathered}
R(x)=\sum_{p=0}^{\infty} G(x)^{p}=\frac{1}{1-G(x)}=\frac{1-x}{1-x e^{1-x}} . \\
R(x) \simeq \frac{2}{1-x}+O(x-1)
\end{gathered}
$$

Therefore,

$$
\lim _{n \rightarrow \infty}\left\langle k_{n}\right\rangle=2 .
$$

\section{References}

[1] B. Baker and R. Shostak, "Gossips and telephones", Discrete Math., vol. 2, pp. 191-193, 1972.

[2] R.T. Bumby, "A problem with telephones", SIAM J. Alg. Disc. Meth. vol. 2, pp. 13-18, 1981.

[3] A. Hajnal, E.C. Milner and E. Szemeredi, "A cure for the telephone disease", Canad. Math. Bull., vol. 15, pp. 447-450, 1976.

[4] T. Tijdeman, "On a telephone problem", Nieuw Arch. Wisk., vol. 3, pp. 188-191, 1971.

[5] A. Seress, "Quick gossiping by conference calls", SIAM J. Disc. Math., vol. 1, pp. 109-120, 1988.

[6] D.B. West, "Gossiping without duplicate transmissions", SIAM J. Alg. Disc. Meth., vol. 3, pp. 418-41, 1982.

[7] D .B. West, "A class of solutions to the gossip problem, part I", Disc. Math., vol. 39, no. 3, pp. 307-326, 1982; "part II", Disc. Math., vol. 40, no. 1, pp. 87-113, 1982; "part III", Disc. Math., vol. 40, no. 2-3, pp. 285-310, 1982.

[8] G. Fertin and A. Respaud, "A survey on Knödel graphs", Discrete Applied Mathematics vol. 137, no. 2, pp. 173-195, 2003.

[9] R. Labahn, "Kernels of minimum size gossip schemes", Discr. Math., vol. 143, pp. 99-139, 1995.

[10] R. Labahn, "Some minimum gossip graphs", Networks, vol. 23, pp. 333-341, 1993.

[11] G. Fertin and R. Labahn, "Compounding of gossip graphs", Networks, vol. 36, pp. 126-137, 2000.

[12] Z. Ho and M. Shigeno, "New bounds on the minimum number of calls in failure-tolerant Gossiping", Networks, vol. 53, pp. 35-38, 2009.

[13] K. A. Berman and M. Hawrylycz, "Telephone problems with failures", SIAM J. Alg. Disc. Meth., vol. 7, pp. 13-17, 1986.

[14] R.W. Haddad, S. Roy and A. A. Schaffer, "On gossiping with faulty telephone lines", SIAM J. Alg. Disc. Meth., vol. 8, pp. 439-445, 1987.

[15] T. Hasunuma and H. Nagamochi, "Improved bounds for minimum fault-tolerant gossip graphs", LNCS, vol. 6986, pp. 203-214, 2011.

[16] L. Gargano, "Tighter time bounds on fault-tolerant broadcasting and gossiping", Networks, vol. 22, pp. 469-486, 1992. 
[17] K. Menger, "Zur allgemeinen Kurventheorie", Fund. Math., vol. 10, pp. 96-115, 1927.

[18] D. J. Daley and D. G. Kendall, "Epidemics and rumors", Nature, vol. 204, 1118, 1964, dx.doi.org/10.1038/2041118a0

[19] R. Karp, C. Schindelhauer, S. Shenker and B. Vocking, "Randomized rumor spreading", In Foundations of Computer Science, Proceedings. 41st Annual Symposium on IEEE, pp. 565-574, 2000.

[20] D. Wilkinson and J. F. Willemsen, "Invasion percolation: a new form of percolation theory", Journal of Physics A: Mathematical and General, vol. 16, no. 14, pp. 33-65, 1983.

[21] R. Chandler, J. Koplik, K. Lerman, J. Willemsen, "Capillary displacement and percolation in porous media", Journal of Fluid Mechanics vol. 119, 249-267, 1982.

[22] A.-L. Barabasi, "Density functional and density matrix method scaling linearly with the Number of atoms", Phys. Rev. Lett., 76, 3750, 1996.

[23] R.C.Prim, "Shortest connection networks and some generalizations", Bell System Technical Journal, vol. 36, no. 6, pp. 1389-1401, 1957.

[24] V. H. Hovnanyan, Su. S. Poghosyan and V. S. Poghosyan, "New methods of construction of fault-tolerant Gossip graphs", IEEE Proceedings of the International Conference on Computer Science and Information Technologies (CSIT'2013), DOI: 10.1109/CSITechnol.2013.6710341.

[25] V.H. Hovnanyan, Su.S. Poghosyan and V.S. Poghosyan, "Method of Local Interchange to Investigate Gossip Problems", Transactions of IIAP of the NAS of RA, Mathematical Problems of Computer Science, vol. 40, pp. 5-12, 2013.

[26] V.H. Hovnanyan, Su.S. Poghosyan and V.S. Poghosyan, "Method of Local Interchange for the Investigation of Gossip Problems: part 2", Transactions of IIAP of the NAS of RA, Mathematical Problems of Computer Science, vol. 41, pp. 15-22, 2014.

[27] V. H. Hovnanyan, Su. S. Poghosyan and V.S. Poghosyan, "Graph Plotter: a Software Tool for the Investigation of Fault-tolerant Gossip Graphs", Proceedings of International Conference CSIT-2013, pp. 20-22.

[28] V. H. Hovnanyan, Su. Poghosyan and V. Poghosyan, "Fault-tolerant Gossip Graphs Based on Wheel Graphs", Transactions of IIAP of the NAS of RA, Mathematical Problems of Computer Science, vol. 42, pp. 43-53, 2014.

[29] V. H. Hovnanyan, Su. Poghosyan and V.Poghosyan, "Open problems in gossip/broadcast schemes and the possible application of the method of local interchange", Proceedings of International Conference CSIT 2015, pp. 73-78.

\section{Submitted 19.06.2019, accepted 26.11.2019.}




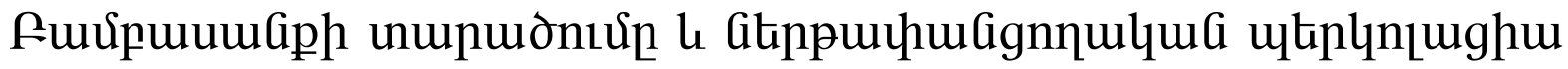

\author{
Unıpta U. Tnnnujua h Ч.uhuqgi U. Tnnnujua

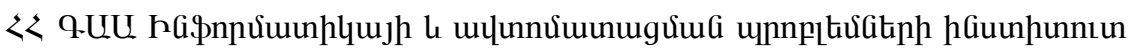 \\ e-mail: psuren55@yandex.ru,povahagn@gmail.com
}

\section{Ư์นnnนnนư}

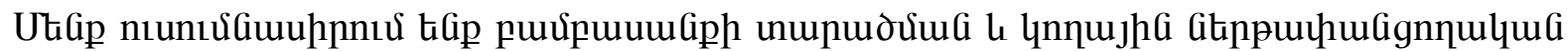

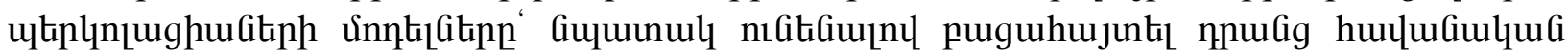

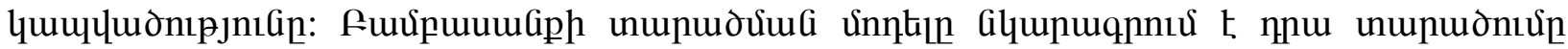

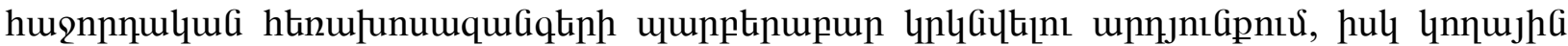

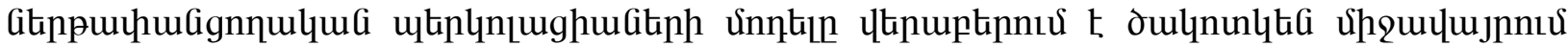

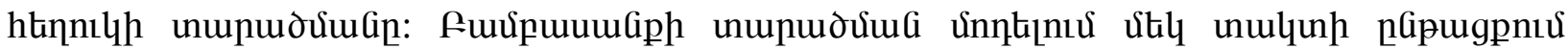

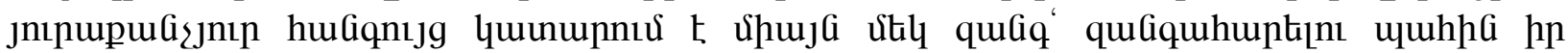

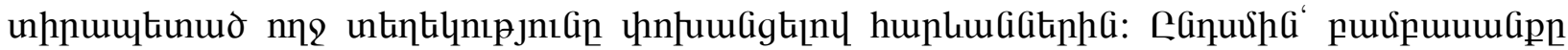

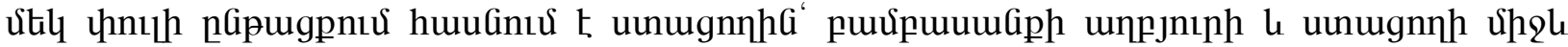

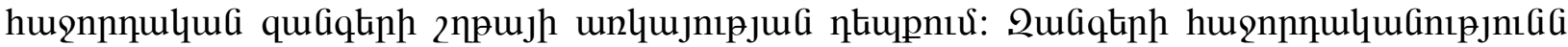

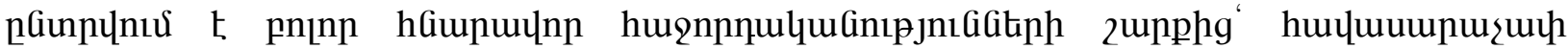

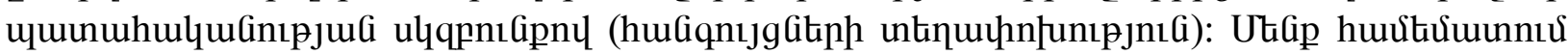

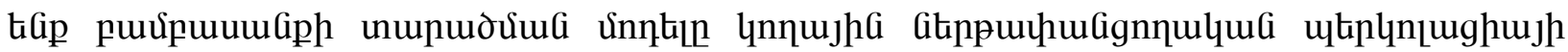

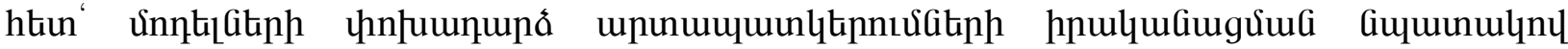

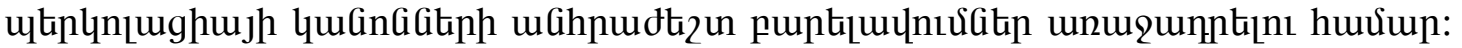

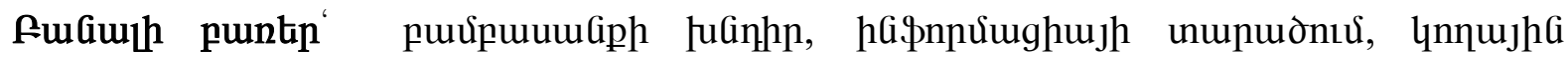

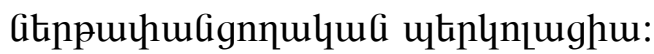

\section{Распространение сплетен и инвазивная перколяция}

\author{
Сурен С. Погосян и Ваагн С. Погосян \\ Институт проблем информатики и автоматизации НАН РА \\ e-mail: psuren55@yandex.ru, povahagn@gmail.com
}

\begin{abstract}
Аннотация
Мы изучаем модели распространения сплетен и реберной инвазивной перколяции с целью обнаружения возможной связи между ними. Модель распространения сплетен описывает распространение как вследствие изза периодического повторения последовательных телефонных звонков, в то время как реберная инвазивная перколяция относится к распространению жидкости в пористой среде. Во время одного раунда распространения слухов каждый узел выполняет вызов только один раз, при этом передавая всю информацию, доступную в момент вызова, своим соседям. Сплетня достигает узла-получателя в течение одного раунда при наличии цепочки последовательных вызовов между источником сплетни и узлом-получателем.
\end{abstract}


Последовательность вызовов выбирается равномерно случайным образом из набора всех возможных последовательностей (перестановок узлов). Мы сравниваем модель распространения сплетен с реберной инвазивной перколяцией с целью выдвижения необходимой оптимизации правил перколяции Аля достижения отображения одной модели на другую, и, наоборот.

Ключевые слова: проблема сплетни, распространение информации, реберная инвазивная перколяция. 